\title{
Écritures de la claustration : le dialogue imaginaire
}

\author{
Marjorie Bertin \\ Université Sorbonne Nouvelle - Paris 3
}

Se retrouver dans un état d'extrême secousse, éclaircie d'irréalité, avec dans un coin de soi-même des morceaux du monde réel.

A. Artaud, Le Pèse-nerfs

Si les écritures testimoniales abondent dans la littérature moderne et contemporaine, elles présentent un intérêt particulier lorsqu'elles sont écrites au cours de l'expérience, et non a posteriori. L'écriture n'est alors plus tentative de reconstruction du passé, avec les apories que la mémoire peut 
rencontrer: l'oubli, la tentation de déformer pour juger, la «mise en intrigue» (Ricœur, 2013, p.311), la tentation du mensonge bien sûr, ou encore notamment cette "obsession commémorative » que dénonce Pierre Nora dans Les Lieux de mémoire (p.977-1012), où il souligne à ce propos que les témoignages et les commémorations deviennent parfois plus importants que les événements eux-mêmes. À contrario, ces écritures testimoniales, prises sur le vif, s'inscrivent dans le présent vécu des auteurs. Les écritures testimoniales de la claustration forcée (exil, asile, prison, hôpital) sont fréquemment, lorsqu'elles le peuvent, écrites au présent et surtout à l'attention d'un destinataire, réel ou imaginaire, comme si l'auteur ne pouvait résister seul à l'expérience qu'il est en train de vivre.

Privé d'autres regards que le sien, le témoin oculaire, sinon sensoriel, devient alors une "institution naturelle », pour reprendre René Dulong (1998), et éprouve la nécessité d'écrire, de décrire son expérience à autrui. En 1925, Antonin Artaud écrit dans Le Pèse-Nerfs que personne, jamais, ne pourra témoigner véritablement de lui et de sa pensée reptilienne, à part lui-même, et d'y poursuivre sur la difficulté, sinon l'impossibilité de témoigner véritablement de soi. Pourtant, vingt ans plus tard, alors qu'il est interné à Rodez (1943), il entreprend d'écrire, de cette « écorce / écriture arrachée au vif d'un corps pensant » (Chaleix, 2003, p. 12-13), à son psychiatre, qu'il voit cependant chaque matin, une cinquantaine de lettres, aujourd'hui rassemblées dans les Nouveaux Écrits de Rodez en compagnie d'autres textes inédits.

Les préoccupations domestiques et les ruses ne sont pas exclues de cette littérature épistolaire, qui semble bel et bien 
permettre à Artaud de résister à la pression de l'internement. L'écriture testimoniale se double ainsi ici d'une fonction refuge, devenant pour l'auteur le dernier rempart contre la folie dans le dialogue imaginaire qu'il instaure avec le lecteur. Ce dialogue apparaît également dans Lejour où Nina Simone a cessé de chanter (2008), de Darina Al Joundi, dans lequel cette dernière, par le biais de la mise en intrigue de sa propre personne en personnage (Darina devient Noun), revient sur sa vie au Liban en se focalisant sur un événement particulièrement traumatique, qui fut le point de départ de l'écriture. Le texte est un parcours rétrospectif depuis l'enfance de l'auteure au milieu de la guerre civile et ses violences jusqu'à son internement en hôpital psychiatrique pour s'être opposée aux intégristes lors des funérailles de son père. Les difficultés sont plus grandes encore que pour Artaud, car contrairement à ce dernier, la comédienne, qui commence à écrire pour résister à la pression de l'internement et de l'isolement, ne jouit pas d'un statut d'écrivain. C'est donc mentalement que Darina Al Joundi, privée de toute possibilité d'écrire ou d'échanger avec quelqu'un, commence le récit qu'elle apprend par cœur de son expérience en hôpital psychiatrique, en écrivant dans les airs à son père, témoin défunt de la claustration.

Pour Antonin Artaud comme pour Darina Al Joundi, le lecteur fait partie intégrante du témoignage et accompagne donc, fictivement, au quotidien l'épreuve endurée. C'est une figure construite avec un destinataire précis: le docteur Ferdière pour Artaud, son père Assem Al Joundi, lui aussi écrivain, pour Darina Al Joundi. Quelles sont les motivations d'un tel dialogue ? Est-ce pour conjurer le risque de devenir l'un de ces nombreux «témoins qui ne rencontrent jamais l'audience capable de les écouter et de les entendre » (p. 207), 
évoqué par Ricœur dans La Mémoire, l'Histoire, l'Oubli? Pourquoi, lorsque les enjeux sont aussi grands, l'écriture n'estelle plus pour soi, tournée vers soi-même mais peut-être plus que jamais pour autrui? Au sein des Nouveaux Écrits de Rodez et du Jour où Nina Simone a cessé de chanter, les auteurs ne trouveraient-ils pas finalement la possibilité de survivre à l'horreur et de résister à la folie grâce à ce dialogue imaginaire, ce voyage immobile avec le lecteur? Quel rôle joue véritablement ce dernier face à la peur et à la solitude de l'auteur? La relation avec le lecteur de ces auteurs, n'est-elle pas ce qui leur permet de dépasser ce «lointain intérieur», pour reprendre le titre d'Henri Michaux, qui les dévore?

Nous verrons ici, partant des cas d'Antonin Artaud et de Darina Al Joundi, comment ce lecteur qui ne répond pas participe à l'écriture, devenue chez ces deux auteurs, à presque cent ans d'intervalle, un processus vital de résistance à l'aliénation.

\section{Une écriture de la claustration}

Les rapports à l'écriture de Darina Al Joundi et d'Antonin Artaud sont foncièrement différents. La première, comédienne, n'avait jamais écrit avant son internement en asile psychiatrique. L'expérience de l'asile, l'Asfourieh au Liban, alors qu'elle est privée de toute possibilité d'écrire et de communiquer, permet la naissance de l'écriture, une écriture mentale dans un premier temps, qu'elle décrit comme un exutoire capital et concret à la folie :

Lorsque j'étais enfermée à l'asile au Liban, on m'a interdit de lire et d'écrire. Et j'ai flippé. Je n'avais jamais écrit avant. Alors 
là-bas, je m'enfermais dans le placard et j'écrivais dans l'air, littéralement. J'écrivais ce que je dis dans le spectacle. Je dis que j'écrivais «Le Liban est un pays démocratique libre ». (2013, p. 104)

Libérée de sa camisole de force mais toujours à l'asile et privée de tout contact avec l'extérieur, Darina Al Joundi se met donc à écrire mentalement à son père une longue lettre dans laquelle elle revient sur les conséquences de l'éduction laïque qu'il lui a donnée. Cette lettre donna lieu à un premier texte publié d'abord sous forme de récit, Le jour où Nina Simone a cessé de chanter, puis à un spectacle éponyme (publié également), monologue qu'elle interprétera seule en scène et qui la révélera au public français.

Le recours à l'écriture pendant la claustration forcée prend une double valeur. En effet, elle permet à Darina Al Joundi non seulement de projeter un témoignage pour le futur, mais aussi de résister à la violence de l'internement en se saisissant du réel :

Quand en 2001 mon père nous a quittés et que j'ai vécu l'atrocité de la violence et de l'enfermement, je ne pensais qu'à une seule chose: à ma sortie de l'asile je ferais tout mon possible pour partir et raconter mon histoire sur scène.

Car dedans, je ne croyais pas ce que j'étais en train de vivre. Je me disais tout le temps: c'est un film, une pièce, c'est à raconter, absolument.

Du coup je m'observais et j'observais ce qui se passait autour de moi avec un œil différent. Chaque détail, chaque couleur, chaque son, chaque odeur, avait un sens différent. (2013, p. 7-8)

La mise en mots permet à terme la réhabilitation du témoin. La résilience passe donc ici à la fois par l'écriture et par la profération. Au cœur de l'internement, c'est par l'exercice 
d'observation et d'écriture mentale que l'auteur parvient à résister à un quotidien insoutenable.

L'écriture épistolaire, chez Artaud, est radicalement différente. Artaud, comme il l'écrit lui-même dans sa première lettre au docteur Ferdière, est autant auteur qu'il est comédien. Sa correspondance littéraire ne débute pas avec son premier internement en 1937. D'autre part, qu'elles soient physiques ou psychiques, les expériences (drogues, délires, souvenirs, psychotropes, relations, etc.) sont la matière organique de son écriture.

Les premières correspondances d'Artaud, écrivain de l'expérience, remontent à 1921 avec Les Lettres et poèmes à Génica Athanasiou (1969 et 2004), une comédienne dont l'auteur du Pèse-nerfs était follement amoureux. Le processus n'est guère différent de celui qu'il emploiera plus de vingt ans après avec le docteur Ferdière. Il n'est pas indispensable d'être loin pour que l'écriture épistolaire s'engage. En effet, alors qu'il lui écrit lettres et poèmes, Artaud partage le quotidien de Génica Athanasiou. Les lettres sont un exutoire aux absences ponctuelles de cette dernière et aux craintes qu'elle génère. L'écriture n'est jamais superflue chez Artaud. Paule Thévenin le confirme dans Antonin Artaud, ce Désespéré qui vous parle (1993) en précisant qu'il était indispensable pour l'auteur de Van Gogh ou le suicidé de la société de n'écrire qu'en ayant "quelque chose d'essentiel à dire mais à condition d'être également capable de savoir que cette chose est essentielle» (p. 68). Les lettres d'Artaud trahissent notamment une véritable terreur de l'abandon. Comme si, perdant l'amour de Génica, perdant par là aussi un témoin de sa vie, Artaud perdrait immanquablement également la raison : 
Et surtout ne parle pas de m'abandonner. Je suis seul au monde, je peux le dire. Je suis environné d'abîmes, encerclé de douleurs. [...] Je serai capable de me suicider immédiatement si tu m'écris une seule fois dans les mêmes termes. Pense à l'angoisse horrible dans laquelle tu viens de me mettre. (p. 57)

Chez Darina Al Joundi comme chez Antonin Artaud, le destinataire du témoignage est capital. Dans les deux cas, le premier lecteur / spectateur est une autorité. Le choix du destinataire dans ce type de relation épistolaire est fondamental, car il devient le dépositaire de la raison du témoin, mise en doute par l'autorité, représentée par des instances psychiatriques qui privent le témoin du regard du monde. Le choix d'une autre autorité vient donc contrebalancer ce poids, avec toute l'ambiguïté que cela comporte et sur laquelle nous reviendrons plus loin, lorsque cette autorité appartient au corps médical concerné, comme chez Artaud. C'est l'autorité paternelle qui est choisie par la comédienne: l'homme qui lui a donné la vie est aussi pour Darina Al Joundi un modèle de laïcité et de liberté. Choisir de lui écrire (par-delà la mort) au cœur de l'acmé, c'est ainsi trouver le destinataire idéal : celui qui ne fera pas de reproches, celui qui ne sera pas malade d'inquiétude et d'impuissance. En un mot, celui auquel il sera permis de tout dire. Dans Le jour où Nina Simone a cessé de chanter, loin de créer une aporie, la mort du destinataire permet ainsi le dévidement de la parole là où la pudeur et la peur de blesser auraient pu être un frein si son père avait été encore en vie: "J'avais le droit de ne pas tout te dire, mais maintenant il faut que tu saches. » (p. 48) Noun / Darina peut maintenant, par exemple, avouer à son défunt père que jeune fille, il lui est arrivé d'être révoltée contre lui au point d'essayer de l'assassiner (p. 48-49). L'écriture testimoniale, dans ce cadre, permet d'établir le bilan d'une vie. Au moment où la raison se 
trouve attaquée, mise en péril dans ses moindres recoins, la rétrospection écrite permet de souligner la cohérence de ces " hasards qu'on ordonne », pour reprendre le titre d'un roman de Hocéïn Faraj. L'analepse, figure par excellence des écritures testimoniales, à l'intérieur de laquelle les événements s'enchaînent chronologiquement, souligne comme une preuve la cohérence de l'existence. Le témoignage se veut le plus vrai possible.

\section{Témoin intime et témoin politique}

Chez Artaud, le choix du destinataire est tout aussi capital, mais la relation très différente. Certes, la véracité et l'objectivité importent peu à l'auteur de l'Ombilic des limbes, mais Ferdière n'est pas un destinataire anodin: en tant que psychiatre d'Artaud dans la clinique où celui-ci se trouve, il a une incidence concrète sur sa vie. C'est pourquoi, en dépit des nombreuses tentatives de réhabilitation du docteur Ferdière par une partie de la critique au cours de ces vingt dernières années, l'écriture est par endroits douloureusement contrainte, attestant ainsi du rapport de force qui sous-tend la relation d'Artaud à son psychiatre. Le lecteur d'Artaud n'a ainsi pas le moindre doute concernant les intentions de l'auteur et la souffrance qu'il endure lorsque ce dernier écrit au docteur Ferdière qu'il veut bien à tout jamais renier une partie de son œuvre, si cela peut lui éviter d'endurer à nouveau la torture des électrochocs :

Quant à la violence de certaines images ou de certains tableaux faites-moi confiance pour mettre cela au point et pour chasser de ma conscience ce qu'il y reste de grossier, de trop vif, de maladroitement audacieux et qui semble revêtir la forme d'une obsession mais qui en réalité n'en est pas une, croyez-moi. Ce 
traitement d'électro-choc m'a fait terriblement souffrir et je vous prie de m'éviter une nouvelle douleur. (2003, p. 73)

Expression du moi pour Artaud, l'écriture s'adresse donc ici à une autorité de chaire, belle et bien présente, qui peut donc influer directement sur les conditions de vie de l'auteur et avec laquelle il est donc, malgré tout, indispensable de prendre certaines précautions.

Il en va autrement dans Le jour où Nina Simone a cessé de chanter. En effet, la pièce de Darina Al Joundi n'a de cesse de montrer à quel point l'histoire personnelle de Noun a été conditionnée, depuis sa plus tendre enfance, par la guerre civile libanaise. L'écriture est alors également la possibilité de se dire autre que soi-même. Ce personnage central de la pièce, qui dit «je» avec les mots et le passé de Darina Al Joundi, est clairement un témoin «politique». Elle s'appelle Noun, mais pourrait tout à fait s'appeler autrement, être autre. L'auteure est parvenue à dédoubler ce personnage d'elle-même. Dans sa Note de présentation au Jour où Nina Simone a cessé de chanter, Darina Al Joundi insiste justement sur l'importance de ce dédoublement, d'autant plus nécessaire, on l'aura compris, qu'elle interprète elle-même son propre rôle en scène. Comme si la création d'un tiers permettait de témoigner tout en se mettant à distance, l'auteur devenant à la fois le rescapé du traumatisme et son conteur :

Avec Noun j'ai créé un personnage de scène. Noun ce n'est pas moi, c'est une de mes Moi. Une moi que j'aime et que la vie, la vraie, et la société ne supportent plus, qui ne lui permettent plus de crier haut et fort ce qu'elle pense, alors qu'au théâtre on l'applaudit. (p. 8)

L'écriture se fait donc testimoniale, moins pour faire état de son parcours intime que pour montrer la violence que l'on peut 
faire subir sans vergogne aux femmes en révolte contre l'obscurantisme. C'est pourquoi son témoignage tend fréquemment vers ce que Jean-Pierre Sarrazac, dans Le Geste de témoigner, Un dispositif pour le théâtre (2011) définit comme «l'Im-personnage» :

L'Im-personnage est le témoin de sa propre vie, non pas individuelle et fixée mais nomade et transpersonnelle. Il est le martyr - martur, en grec, signifie témoin. Un témoin, c'est-àdire, comme chez Brecht, un tiers, qui témoigne non plus pour le Christ mais pour l'Homme. (p. 18)

De fait, en se dédoublant, Darina Al Joundi devient le témoin, pour les autres, de sa propre histoire, cette dernière n'étant alors plus seulement douleur personnelle mais exemple pour les autres. Le témoin est donc chez elle, viscéralement, un témoin politique.

Artaud appartient quant lui à un autre type de témoin poussé à son extrême : le témoin intime. Ainsi, dans Nouveaux Écrits de Rodez, pourtant rédigés entre 1943 et 1946, la place accordée à la Seconde Guerre mondiale est-elle quasiment inexistante et toujours en lien avec la propre individualité d'Artaud. Ce dernier, concentré sur lui-même et sur l'exploration de la folie, ne semble guère préoccupé par la question politique. Comme en témoignent L'Ombilic des limbes mais aussi Pour en finir avec le jugement de Dieu, l'écriture du « je » est ce qui permet au « je » d'Artaud, projeté dans un corps étranger et "séparé » de lui-même, comme il l'a écrit dans Les Nouvelles Révélations de l'Être, de rester en vie.

Lorsqu'il écrit le réel - presque toujours lorsqu'il y est contraint - , celui-ci ne lui inspire guère que de la défiance, car Artaud croit quant à lui au surréel : 
Et la surréalité est comme un rétrécissement de l'osmose, une espèce de communication retournée. Loin que j'y voie un amoindrissement du contrôle, j'y vois au contraire un contrôle plus grand, mais un contrôle qui, au lieu d'agir, se méfie, un contrôle qui empêche les rencontres de la réalité ordinaire et permet des rencontres plus subtiles et raréfiées, des rencontres amincies jusqu'à la corde, qui prend feu et ne se rompt jamais. (1968 [2012], p. 88)

\section{Artaud, le magicien}

Artaud / Nalpas - il signe sous ces deux patronymes (Nalpas est le nom de sa mère) durant son internement à Rodez - est foncièrement auteur, comédien et metteur en scène, donc magicien. Son écriture est vivante. Il est du domaine des songes, de l'illusion, du monde imaginaire réalisé à travers la scène et la mise en mots. Ainsi n'est-ce guère surprenant si, lorsqu'il se plaint des traitements qui lui sont infligés, c'est naturellement du côté de la magie, en lançant sorts et menaces au docteur Ferdière, qu'il cherche refuge :

Il n'aurait pas fallu me faire de l'électro-choc [...] parce que ce faisant vous m'avez démagnétisé donc mis en état de moindre résistance devant les assauts des forces pernicieuses qui nous guettent tous et qui sont en cause de toutes nos maladies tant mentales que physiques en attaquant d'abord notre cerveau et notre système sympathique et nerveux. Et mon cher ami occultement tous les êtres de même race se tiennent, tous ceux qui s'aiment sont liés occultement, me faire du mal à moi c'est vous faire du mal à vous et réciproquement [...]. (1943, p. 889-890)

L'écriture d'Artaud, témoin et champ d'expérience de lui-même par excellence jusqu'à la fin de sa vie, est à la fois intime et unique. Aussi contraignant soit-il, l'internement est ainsi régulièrement pulvérisé, atomisé par la création. En effet, si les 
épreuves quotidiennes de l'internement sont bel et bien présentes dans son texte, Artaud parvient souvent, lorsqu'elles n'entravent pas trop sa vie, à les dépasser pour créer un discours poétique et mystique dont il est le seul Conteur possible. Et la folie, écrit-il au docteur Ferdière dans une lettre aux accents rimbaldiens, n'a rien à voir dans ce processus, propre à l'artiste et inhérent à toute création :

C'est de mon moi profond que je tire mes poèmes et mes écrits et vous les aimez. Tout poète est un Voyant. C'est de son illuminisme que Rimbaud a tiré les Illuminations et la Saison en Enfer. Et William Blake avait vu dans le monde mystique de l'Esprit l'objet de toutes les visions merveilleuses transcrites dans le Mariage du Ciel et de l'Enfer. Si je ne croyais pas dans les images mystiques de mon cœur je ne pourrais pas arriver à leur donner la vie. (2003, p. 96)

Le père de Darina $\mathrm{Al}$ Joundi lui a donné la vie et une éducation. Le docteur Ferdière, même s'il a probablement sauvé la vie d'Antonin Artaud en acceptant son internement à Rodez ${ }^{1}$, est le psychiatre de la clinique dans laquelle il se trouve enfermé et lui fait subir des traitements qui le font considérablement souffrir. Le destinataire des lettres (plusieurs dizaines) est donc également le geôlier de leur auteur. La liberté de l'auteur dépend du docteur Ferdière, psychiatre de son état. En lui écrivant des lettres comme "L'Hymne aux Daimons », réflexions issues de sa lecture de Ronsard, Artaud prouve également qu'il recouvre ses facultés intellectuelles, que ses

\footnotetext{
1 «Antonin Artaud aura passé neuf ans dans divers asiles d'aliénés, à une époque où les conditions de vie, ou de survie des malades y étaient particulièrement dramatiques. [...] On s'accorde aujourd'hui à reconnaître que son départ de Ville-Évrard pour l'asile de Rodez a sans doute sauvé Artaud de la chronicisation, cet enlisement définitif dans la vie asilaire qui guette à long terme tant d'enfermés. » Evelyne Grossman, "Préface », dans Artaud, CEuvres, p. 12-13.
} 
démons s'éloignent. L'écriture épistolaire lui permet également de dénoncer les séances d'électrochocs en montrant combien ces dernières lui sont néfastes :

L'électro-choc [...] me désespère, il m'enlève la mémoire, il engourdit ma pensée et mon cœur, il fait de moi un absent qui se connaît absent et se voit pendant des semaines à la poursuite de son être, comme un mort à côté d'un vivant qui n'est plus lui, qui exige sa venue et chez qui il ne peut plus entrer. (2004, p. 1759)

Dans Le jour où Nina Simone a cessé de chanter comme dans Les Nouveaux Écrits de Rodez, le destinataire est comme instinctivement choisi. Le père comme le médecin représentent un auditoire intime qui peut entendre beaucoup. De fait, les limites de la bienséance, la norme sociales sont abolies. La sincérité vient déborder de cette langue qui, chez Artaud, finit parfois par s'étrangler sur les humiliations et les traumatismes du quotidien qui viennent contaminer l'écriture : «il y a deux mois que vous m'avez promis de m'envoyer une brosse à dents, vous ne l'avez pas fait» (p. 36), écrit Artaud, désespéré de l'insalubrité de l'asile au docteur Ferdière.

\section{Le témoin compagnon de l'éternité}

L'écriture de soi permet également de fixer celui qui écrit dans le temps en lui offrant la temporalité de l'œuvre d'art, cherchant à atteindre le chœur de l'humain lorsqu'il lui semble que la crise n'est pas supportable. Car, comme le dit Marguerite Yourcenar à propos de Feux, son recueil de poèmes en prose sur la passion, "Écrire c'est déjà dominer une crise et la surmonter. » Le seul moyen de résister, pour Artaud prisonnier de son corps et sentant la folie qui le guette, est de s'inscrire dans l'éternité de l'œuvre d'art par le témoignage de son 
expérience via son écriture vivante, et ce, d'autant plus que le dualisme pirandellien de la fuite infinie de la vie conditionnée par le corps humain (dualisme vie / forme) est la préoccupation centrale et insoutenable d'Artaud, qui depuis les électrochocs subis au cours de son précédent internement, se considère comme mort les premiers mois de sa vie à Rodez :

Antonin Artaud est mort à la peine et de douleur à Ville-Évrard au mois d'août 1939 et son cadavre a été sorti de Ville-Évrard pendant une nuit blanche comme celles dont parle Dostoïevski et qui occupent l'espace de plusieurs journées intercalaires mais non comprises dans le calendrier de ce monde-ci quoi (que) vraies comme le jour d'ici. (p. 28)

À l'instar de Darina Al Joundi devenue Noun (dont on apprendra qu'il s'agit d'un surnom hérité de l'enfance), Artaud se voit lui aussi contraint de prendre un nom d'emprunt, également puisé dans son passé. Ainsi, comme nous l'évoquions en amont, au début de son internement à Rodez, ne signe-t-il plus ses textes Antonin Artaud mais Antonin Nalpas : Nalpas est le nom de jeune fille de sa mère. Le rejet du patronyme durera longtemps: d'août 1939 à juillet 1943. Après cela, Artaud reprendra son nom patronymique, sous lequel, jusqu'à sa mort, il signera ses textes. Dans sa Présentation aux Nouveaux Écrits de Rodez, Pierre Chaleix met en exergue l'importance de l'écriture comme unique point de réconciliation / conciliation possible entre l'esprit d'Artaud et son corps :

L'angoisse ancienne, l'angoisse de toujours pour Antonin Artaud, c'est la difficulté qu'éprouve l'être à se saisir de sa pensée authentique, la difficulté d'être ce que je suis, eût-il pu dire $-s$ 'il ne l'a pas dit. Car la réalité vraie de l'être coïncide avec cette pensée qui échappe si profondément aux conditionnements extérieurs et aux interdits qu'elle se dérobe à son propre regard. (p. 12-13) 
Comme chez Darina Al Joundi, qui se fictionnalise pour l'écriture en créant le personnage de Noun, Artaud, dans les Nouveaux Écrits de Rodez, témoigne donc lui aussi d'un dédoublement en se définissant comme déjà mort. Être mort, c'est ne plus être soumis au flux continuel et mouvant de l'existence. Mort, l'auteur ne pourra plus récuser, argumenter : de fait, il ne peut plus être attaqué. Dés lors, l'écriture n'est plus empêchée et devient une arme pour résister. Échappant à ce conditionnement, Artaud peut rejoindre d'autant plus facilement l'a-temporalité magique et éternelle de l'œuvre d'art qui toujours survit à son créateur.

Paul Ricœur, dans La Mémoire, l'Histoire, l'Oubli, expose une théorie selon laquelle les écritures testimoniales - et leur critique - sont les seuls véritables moyens d'accréditer «la représentation historienne du passé» (p.364), de ne pas mettre à distance cette dernière en dépit des années et des mutations de la société. Cette théorie s'applique dans le cadre de nos deux objets d'étude, car au-delà du simple témoignage intime et de leur portée littéraire, des textes comme Le jour où Nina Simone a cessé de chanter et les Nouveaux Écrits de Rodez sont aussi, il importe de ne pas l'oublier, de véritables documents historiques et sociologiques concernant les asiles psychiatriques. Darina Al Joundi et Antonin Artaud sont de «vrais témoins». En amont de leur publication / représentation, ces deux textes de "l'écrire vivre», pour reprendre l'expression de Marina Tsvetaieva - autre témoin qui se définissait comme une «sténographe de la vie»-, produits à près de cent ans d'intervalle, montrent également bel et bien à quel point l'écriture et la création sont les derniers remparts contre la folie, les derniers territoires de ceux que l'on a dépossédés de tout. L'écriture testimoniale chez Artaud et 
Al Joundi est donc à la fois manifestation de la trace et exutoire au présent vécu où l'acteur de la claustration devient également spectateur de lui-même dans une ultime tentative de résistance à la folie et à la pression sociale.

\section{Bibliographie}

Al Joundi, Darina. (2013), Le jour où Nina Simone a cessé de chanter, Paris, L'avant-scène théâtre.

ARTAUD, Antonin. (1968 [2012]), L'Ombilic des Limbes suivi de Le Pèse-nerfs, Paris, Gallimard, coll. «Poésie/Gallimard».

-. (2004), CEuvres, Paris, Gallimard, coll. « Quarto ».

-. (2003), Nouveaux Écrits de Rodez, Paris, Gallimard, coll. « L'Imaginaire ».

—. (1969), Lettres à Génica Athanasiou, précédé de Deux poèmes à elle dédiés, Paris, Gallimard, coll. « Le Point du Jour ».

Bertin, Marjorie. (2014), «L'expérience chez Jean Genet: moteur de la création artistique », dans Catherine Naugrette, Le Souci du monde, Paris, L'Harmattan, à paraître.

BousseyrouX, Nicole. (2006), «La passion d'Antonin Artaud», $L^{\prime} e n-j e ~ l a c a n i e n, \mathrm{n}^{\circ} 7$, p. 125-133.

ChaleiX, Pierre. (2003), «Présentation », dans Antonin Artaud, Nouveaux Écrits de Rodez, Paris, Gallimard, coll. «L'imaginaire », p. 12-13.

DulONG, René. (1998) Le Témoin oculaire. Les conditions sociales de l'attestation personnelle, Paris, EHESS. 
FARAJ, Hoceïn. (1999), Ces hasards qu'on ordonne, Paris, L'Harmattan.

NoRA, Pierre. (1992), Les Lieux de mémoire, t. 3, Paris, Gallimard, coll. «Bibliothèque des idées ».

Riçeur, Paul. (2000), La Mémoire, l'Histoire, l'Oubli, Paris, Seuil, coll. « L'ordre philosophique».

SARRAZAC, Jean-Pierre. (2011), "Le Témoin ou le Rhapsode ou Le Retour du Conteur», Études théâtrales, n51-52, p. 13-25.

Thevenin, Paule. (1993), Antonin Artaud, ce désespéré qui vous parle, Paris, Seuil, coll. « Essais ».

Tsvetaieva, Marina. (2009), OEuvres, t.I: Prose autobiographique, Paris, Seuil.

YOURCENAR, Marguerite. (1979), entretien avec Jacques Chancel, Radioscopie, $<$ http://boutique.ina.fr/audio/art-etculture/litterature/PACK734275808/margueriteyourcenar.fr.html>.

\section{Résumé}

Si les écritures testimoniales abondent, rares sont celles qui sont écrites au cours de l'expérience, et non a posteriori. L'écriture n'est alors plus tentative de reconstruction du passé, mais témoignage du présent. Ce type d'écriture apparaît surtout dans les écritures de la claustration, comme si l'auteur ne pouvait résister seul. Épistolaire, l'écriture testimoniale se double ainsi d'une fonction refuge, devenant pour l'auteur le dernier rempart contre la folie. Avec les Lettres d'Antonin Artaud au docteur Ferdière (1943) et Le Jour où Nina Simone a cessé de chanter (2008) de Darina Al Joundi, nous montrerons en quoi ces écritures épistolaires sont des marques de résistance à la claustration. 


\begin{abstract}
If testimonials are abundant in modern literature, and written $a$ posteriori, very few of them were actually written during the experience. In the latter case, writing is not an attempt to reconstruct the past, but rather a witness' account of the present. This type of writing prevails mostly when the author is confined, as if he/she were unable to cope with the situation alone. Writing testimonials becomes then a refuge; the last bastion against insanity. With Lettres d'Antonin Artaud au docteur Ferdière (1943) and Le Jour où Nina Simone a cessé de chanter (2008) by Darina Al Joundi, I will study how these writings are signs of resistance to confinement.
\end{abstract}

